\title{
The Klf6-related super enhancer regulates KIf6-SV2 expression mediated proliferation in human hepatoma (HepG2) cells
}

\author{
Kum Chol Ri ${ }^{1,2^{*}}$, Chol Kim ${ }^{1,2}$, Sun $\|$ Choe ${ }^{1,3}$, Ju Hyok So ${ }^{4}$ and Se Hyok $\mathrm{O}^{1}$
}

\begin{abstract}
Background: The Klf6 gene, which belongs to Krüppel-like family of $\mathrm{C} 2 \mathrm{H} 2$ zinc finger transcription factors, is greatly related to tumorigenesis via a high rate of somatic mutation in the carcinomas of prostate, liver, colon, stomach, lung, neck, pituitary, and nervous system: Furthermore, the pathways regulating the expressions of Klf6 splice variants termed KIf6-SV1, -SV2, and -SV3 remain obscure although their functional outcomes have been clear. In this study, the functional roles of Klf6 variants in the inhibition of cell proliferation induced by the disruption of Klf6related super enhancer in human hepatoma (HepG2) cells were evaluated.

Results: As a result, the disruption of Klf6-related super enhancer not only induced the upregulation of KIf6-SV2 but also led to a significant reduction of proliferation in HepG2 cells. In addition, the disruption of Klf6-related super enhancer led to the induction of p21 and Bax genes mediated by the upregulation of KIf6-SV2.

Conclusion: In conclusion, it was demonstrated that Klf6-related super enhancer modulates cell proliferation via the regulation of KIf6-SV2 expression in human hepatoma (HepG2) cells. The results provide the functional significance of KIf6-related super enhancer in understanding the transcriptional regulation mechanism of Klf6.
\end{abstract}

Keywords: Super Enhancer, KIf6 gene, Genome editing, CRISPR, KIf6 isoforms

\section{Background}

Liver cancer is one of the most common six malignant tumors with high malignancy and a poor prognosis around the world. Although the genetic information and the risk factors of liver cancer formation have been increasingly identified, the exact mechanisms underlying the initiation, development, and metastasis of liver cancer are poorly understood [1].

The Klf6 gene, which belongs to Krüppel-like family (containing $\mathrm{C} 2 \mathrm{H} 2$ zinc finger transcription factors), is greatly related to tumorigenesis via a high rate of somatic mutation in the carcinomas of prostate, colon, stomach, liver, lung, neck, pituitary, nervous system, etc. $[2,3]$. The functional role of dysregulated alternative splicing in cancers is now recognized in a range of human cancers including liver, lung, stomach, prostate,

\footnotetext{
* Correspondence: rkc19810303@126.com; RKCHIT@hit.edu.cn

${ }^{1}$ School of Management, Harbin Institute of Technology, Harbin, China

${ }^{2}$ Life Science Department, University of Science, Pyongyang, Democratic

People's Republic of Korea

Full list of author information is available at the end of the article
}

colon, etc. It has been reported that many genes associated with cancer can be alternatively spliced, and their expression can lead to the production of multiple splice variants with antagonistic function. Generally, the Klf6 gene, which is known to be an important gene in the initiation, development, and metastasis of tumors, can be alternatively spliced into biologically active isoforms called Klf6-SV1, $-S V 2$, and -SV3 $[4,5]$. In particular, the $S V 1$ variant has been shown to accelerate the progression and metastasis of prostate cancer $[6,7]$. The functional role of Klf6-SV1 in tumorigenesis was most well-defined among three Klf6 spliced variants because it can be highly expressed in many human malignant tumors, and its over-expression is observed in cancer patients with poor prognosis and low survival rate [8-10]. Growing results have shown that elevated expression of Klf-SV1 variant partially promotes the metastasis, progression, and mortality of cancer through its antagonism to Klf6 [11]. Furthermore, elevated Klf6-SV1/Klf6 mRNA ratios have been demonstrated with their functional characteristics in liver cancer samples. On the other hand, 
over-expression of $S V 2$ variants in IHH and HepG2 cells was observed to significantly reduce cell proliferation by apoptosis [12-14]. Although the functional outcome of Klf6 splice variant has become clear, the pathway regulating expression of Klf6 splice variant remains ambiguous.

Recently, a large cluster of transcriptional enhancers called super enhancers (SEs) have been identified. Furthermore, SEs have been shown to specifically regulate cell identity in many tumors, including liver, lung, stomach, and prostate, as genomic domains that are large non-coding enhancers [15-20]. We have already identified a Klf6-related super enhancer in human HepG2 cells using genomic editing techniques and demonstrated that Klf6related SE regulates cellular proliferation as a potent regulator of Klf6 gene expression [21]. We assumed that regulation of cell proliferation by Klf6-related super enhancer may be associated with Klf6 variants in deeper study.

In this study, we obtained the cell clones of a Klf6-related super enhancer deleted by means of the CRISPR/Cas9 system-mediated genetic engineering as previously described [21] and also found that inhibition of cell proliferation by deletion of Klf6-related super enhancer is caused by Klf6 variants in human hepatoma (HepG2) cells. At first, it was demonstrated that deletion of Klf6-related super enhancer induces the upregulation of $S V 2$ variant and leads to the inhibition of cell proliferation in HepG2 cells. In addition, it was shown that Klf6-related super enhancer leads to the induction of $p 21$ (cell-cycle-controlling gene) and $\operatorname{Bax}$ (pro-apoptotic gene) genes mediated by upregulation of SV2 variant. The results provide the functional significance of Klf6-related super enhancer in understanding the transcriptional regulation mechanism of Klf6.

\section{Methods and materials}

\subsection{Cell culture and transfection experiments}

HepG2 was derived from the Chinese Academy of Science. The cell culture and transfection were carried out according to cell culture and transformation protocols as previously described [21].

\subsection{RNA extraction, reverse transcription, and real-time PCR}

Total RNA isolation was performed using TRIzol (Life Tech) extraction for the target clones.

A total of RNA $(1 \mu \mathrm{g})$ was reverse transcribed using the first-strand complementary DNA synthesis with random primers. RT-PCR was performed with KLF6 $5^{\prime}$ and $3^{\prime}$ untranslated region-specific primers on 10 ng cDNA derived from the target clones as previously described [9]. qRT-PCR was carried out with SYBR
Green PCR Master Mix (Applied Biosystems). The relative expression ratio of genes in the cells was quantified by the $2^{-\triangle \Delta C T}$ method.

Primers selected for the experiments are as following: 5'-CGGACGCACACAGGAGAAAA-3' $/ 5$ '-CGGT GTGCTTTCGGAAGTG-3' (Klf6), 5' -CCTCGCCAGG GAAGGAGAA-3'/5' -CGGTGTGCTTTCGGAAGTG3' (Klf6-SV1), 5' -TCGGGGAAGCCAGGAGAA-3'/5' CGGTGTGCTTTCGGAAGTG-3' (Klf6-SV2), 5' CGGACGCACACAGGTGTT-3'/5' -TCTGCTCCCT CAGAGGTGCC-3' (Klf6-SV3), 5'-ACTCTCAGGGTC GAAAACGG-3' $/ 5^{\prime}$-CCTCGCGCTTCCAGGACTG-3' (P21), 5' -ATCCAGGATCGAGCAGGGCG-3' $/ 5^{\prime}$ ACTCGCTCAGCTTCTTGGTG-3' (Bax), 5'-GTGT GGCGACATATGCAGCT-3'/5' -CAAGATCAGCAG TCTCATTC-3' (beta-actin).

The analysis proceeded in triplicate, and the relative level of gene expression was assessed as beta-actin.

\subsection{Cell proliferation assay and Western blot assay}

Proliferation was determined by estimating $[3 \mathrm{H}]$ thymidine incorporation as previously described [9]. Separation of proteins for Western blot assays proceeded on 10-15\% SDS-PAGE gel as previously described [21]. The beta-actin protein was used as a loading control.

\subsection{Plasmid construction and transfection}

The pSUPER-siSV1, -siSV2, and -siSV3 plasmids used to downregulate Klf6-SV1, $-S V 2$ and -VS3 expression were constructed according to the previously described method, and the pSUPER-Luc construct was used as a control [9]. The pSUPER-si-wtKLF6 construct was generated as previously described using the PSUPER vector [22]. To insert the targeting sequence, DNA oligonucleotides were designed and cloned into the BglII-HindIII sites of the pSUPER vector. The characteristics of the designed DNA oligonucleotides are shown in Table 1.

Generation of cell clones over-expressing Klf6-SV variants was performed as previously described [12]. The coding sequences of Klf6 and Klf6-SV variants were obtained by PCR amplification and inserted into p3XFLAG-CMV-10 vector (Sigma).

\section{Results}

3.1 Deletion of KIf6-related SE induces inhibition of cell proliferation in human HepG2 cells

A clone of Klf6-related SE deletion (SE-del) clone was obtained using the CRISPR/Cas9 system to evaluate the effect of Klf6-related SE on the expression of Klf6 gene in HepG2 cells. The experimental procedure for obtaining SE-del clones in HepG2 cells has been detailed in previously published literature [21]. Expression of Klf6 gene in SE-del clone using 
Table 1 DNA oligonucleotides for pSUPER vector

\begin{tabular}{|c|c|c|c|}
\hline $\begin{array}{l}\text { KIf6 } \\
\text { siSV1-F }\end{array}$ & $\begin{array}{l}\text { GATCCCCAGGCTTTTCTCCTTCCCTGGC } \\
\text { ttcaagagaGCCAGGGAAGGAGAAAAGCCTITTGGAAA }\end{array}$ & $\begin{array}{l}\text { KIf6 } \\
\text { siSV1-R }\end{array}$ & $\begin{array}{l}\text { AGCTITCCAAAAAGGCTITCTCCTTCCCTG } \\
\text { GCtctcttgaaGCCAGGGAAGGAGAAAAGCCTGGG }\end{array}$ \\
\hline $\begin{array}{l}\text { KIf6 } \\
\text { siSV2-F }\end{array}$ & $\begin{array}{l}\text { GATCCCCGCCAGGAGAAAAGCCTT } \\
\text { ACttcaagagaGTAAGGCTITTCTCCTGGCTTाTTGGAAA }\end{array}$ & $\begin{array}{l}\text { Klf6 } \\
\text { siSV2-R }\end{array}$ & $\begin{array}{l}\text { AGCTTTCCAAAAAGCCAGGAGAAAAGCCTTA } \\
\text { CtctcttgaaGTAAGGCTTITCTCCTGGCGGG }\end{array}$ \\
\hline $\begin{array}{l}\text { KIf6 } \\
\text { siSV3-F }\end{array}$ & $\begin{array}{l}\text { GATCCCCGCAAGCAGACGAGCCTC } \\
\text { ACttcaagagaGTAAGGCGTATCTCGTGGCTIIITGGAAA }\end{array}$ & $\begin{array}{l}\text { Klf6 } \\
\text { siSV3-R }\end{array}$ & $\begin{array}{l}\text { AGCTTाTCCAAAAAGCGAAGAGACAGGCCTTA } \\
\text { CtctcttgaaGCCAGACTITICTCCTGGCGGG }\end{array}$ \\
\hline Luc-F & $\begin{array}{l}\text { GATCCCCTACTTCGAAATGTCCGT } \\
\text { TCttcaagagaGAACGGACATTTCGAAGTATTITTGGAAA }\end{array}$ & Luc-R & $\begin{array}{l}\text { AGCTITTCCAAAAATACTTCGAAATGTCCGTT } \\
\text { CtctcttgaaGAACGGACATTTCGAAGTAGGG }\end{array}$ \\
\hline $\begin{array}{l}\text { Si- } \\
\text { wtKLF6- } \\
\text { F }\end{array}$ & $\begin{array}{l}\text { GATCCCCTGGCGATGCCTCCCCCGACttcaagaga } \\
\text { GTCGGGGGAGGCATCGCCATTITTGAAA }\end{array}$ & $\begin{array}{l}\text { Si- } \\
\text { wtKLF6- } \\
\text { R }\end{array}$ & $\begin{array}{l}\text { AGCTITTCCAAAAATGGCGATGCCTCCCCCGAC } \\
\text { tctcttgaaGTCGGGGGAGGCATCGCCAGGG }\end{array}$ \\
\hline
\end{tabular}

qRT-PCR was decreased by $80 \%$ or more, and strong inhibition of Klf6 gene expression was observed as shown in Fig. 1a.

Reduced expression of Klf6 gene in SE-del clone was confirmed at the protein level by using Western blot, and expression of Klf6 protein was also significantly inhibited in SE-del clone as compared with the control (Fig. 1b). After deletion of Klf6-related $\mathrm{SE}$, the effect of cell proliferation was evaluated using $[3 \mathrm{H}]$ thymidine incorporation assay, concurrent with the result of decreased Klf6 expression, it observed a markedly inhibited proliferation (Fig. 1c).

\subsection{Deletion of KIf6-related SE regulates expression of KIf6-SV variants in human HepG2 cells}

As shown in the above results, since significant inhibition of cell proliferation as well as expression of $K l f 6$ after deletion of Klf6-related SE was observed, therefore, it attempted to analyze the expression characteristics of Klf6 variants by RT-PCR.

The SE-del clone expression pattern through RTPCR of cDNA derived from the target clone containing the SE-del clone, and the control clone revealed a strong inhibition of the full-length tumor suppressor Klf6 concurrently with a severe change in the

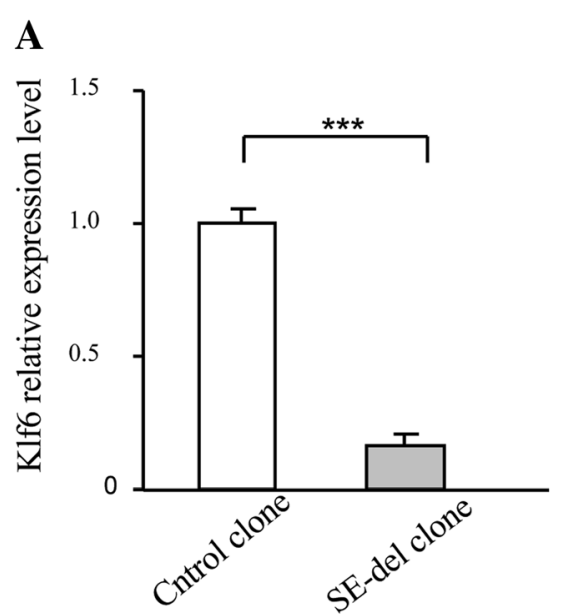

C
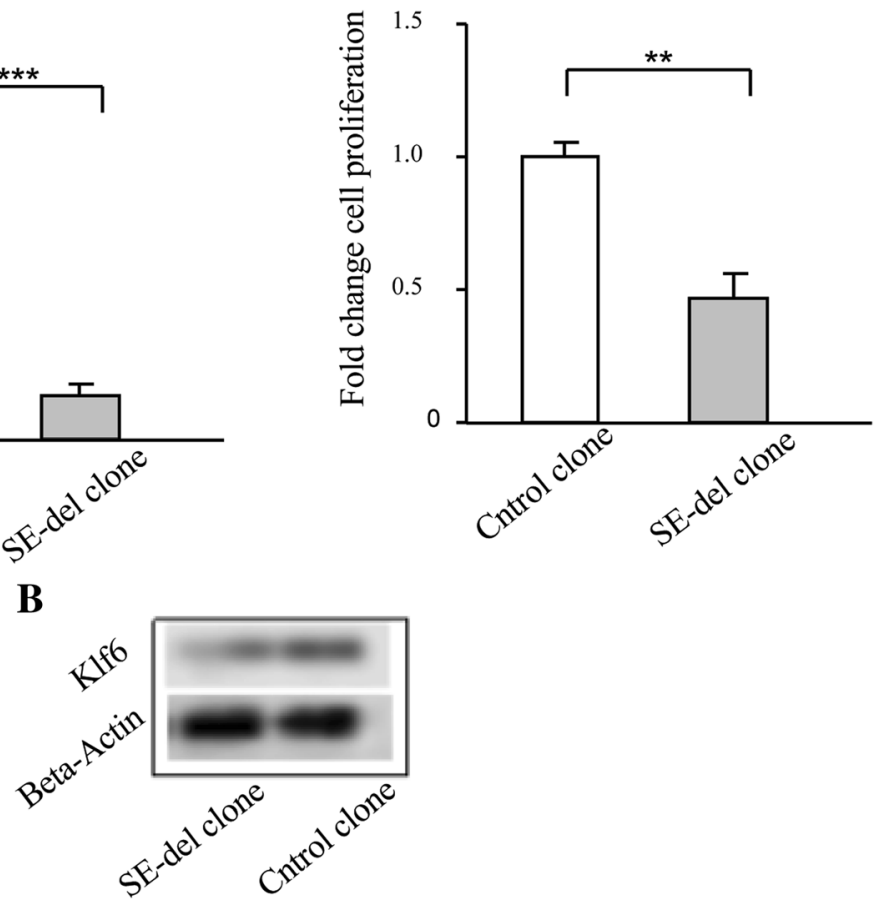

Fig. 1 Klf6 gene expression and cell proliferation assay. a Klf6 gene expression assay. The Klf6 expression level was measured by qRT-PCR. The results represent the mean of five amplifications, ${ }^{* * *} p<0.001$. b Klf6 proteins expression assay. KIf6 proteins were determined by western blot. $\mathbf{c}$ Cell proliferation assay. [3H] thymidine incorporation was determined at $72 \mathrm{~h}$. The results represent the mean of eight experiments, ${ }^{* *} p<0.01$ 
relative expression level of the Klf6 splice variants Klf6-SV1, $-S V 2$, and -SV3 (Fig. 2a). Quantitative realtime PCR (qRT-PCR) analysis of cDNA derived from the target clones showed that the SV/Klf6 mRNA ratio was increased in Klf6-SV1 and Klf6-SV2, but decreased in Klf6-SV3. Especially, the expression of Klf6-SV2 was three times higher than that of the control, but the expression of Klf6-SV1 was increased very little. These results show that expression of Klf6 splice variants in human HepG2 cells is strongly regulated by Klf6-related super enhancer, and in particular, inhibition of cell proliferation after deletion of SE is likely to be associated with overexpression of Klf6-SV2.

\subsection{Deletion of Klf6-related SE induces inhibition of proliferation through over-expression of Klf6-SV2 in human HepG2 cells}

On the basis of the above findings, we conducted an experiment to determine whether over-expression of Klf6-SV2 induced by the disruption of the Klf6-related $\mathrm{SE}$ is actually associated with cell proliferation in human HepG2 cells. Firstly, we specifically inhibited the expression of Klf6-SV1 and $-S V 2$, which showed increased expression after the disruption of the Klf6related SE using target-specific siRNAs in SE-del clone (Fig. 3). Targeted reduction of Klf6-SV1 and $-S V 2$ expression in SE-del clone was confirmed at the mRNA level by qRT-PCR (Fig. 3a). Comparing to the siLuc control cells, the expression level of Klf6-SV1 in the siSV1 cells was decreased by at least $45 \%(p<$ 0.01 ), whereas the expression level of Klf6-SV2 in the siSV2 cells was decreased by at least $55 \%(p<0.05)$. The effects of Klf6-SV1 and $-S V 2$ inhibition were determined by measuring cell proliferation (Fig. 3b). Cell proliferation was increased twofold in siSV2 cells $(p<0.01)$ but decreased by half in siSV1 cells $(p<$ 0.01 ). The results indicate that over-expression of Klf6-SV1 promotes cell proliferation, but its downregulation induces inhibition. On the other hand, overexpression of Klf6-SV2 inhibits cell proliferation, but its down-expression promotes cell proliferation and thus appears to be responsible for inhibition of cell proliferation induced by deletion of the Klf6-related SE.

Secondly, we inhibited the expression of Klf6-SV1, $-S V 2,-S V 3$, and wtKlf6 using target-specific siRNAs in SE-non-del clone (Fig. 3). As shown in Fig. 3c, d, downregulation of Klf6-SV1 $(35 \%, p<0.05)$ induces inhibition of cell proliferation, but down- regulation of Klf6-SV2 $(35 \%, p<0.05)$ promotes cell proliferation. Expression of Klf6-SV3 in siSV3 cells was decreased by at least $72 \%$ $(p<0.05)$ compared to the siLuc control cells, but there was no significant difference in cell proliferation analysis $\left({ }^{\#} p>0.05\right)$. However, expression of the wtKlf6 in siwtKlf6 cells was decreased by $55 \%(p<0.01)$ but a significant increases in cell proliferation $(p<0.01)$. As shown above, the results show that over-expression of Klf6-SV2 induced by the disruption of the Klf6-related $\mathrm{SE}$ is an important factor associated with cell proliferation in human HepG2 cells, especially inhibition of cell proliferation.

\subsection{Over-expression of Klf6-SV2 induces an increased expression of p21 and Bax in human HepG2 cells}

The expression of cell cycle-regulated genes were analyzed to elucidate the proliferation-related mechanism induced by Klf6-SV2. In response to over-expression of Klf6-SV2, cell cycle-regulating $p 21$ (CIP/WAF1) expression was higher about two-fold in SE-del clone than that of control clone (Fig. 4a). Elevated expression in $p 21$ (CIP / WAF1) protein was also identified

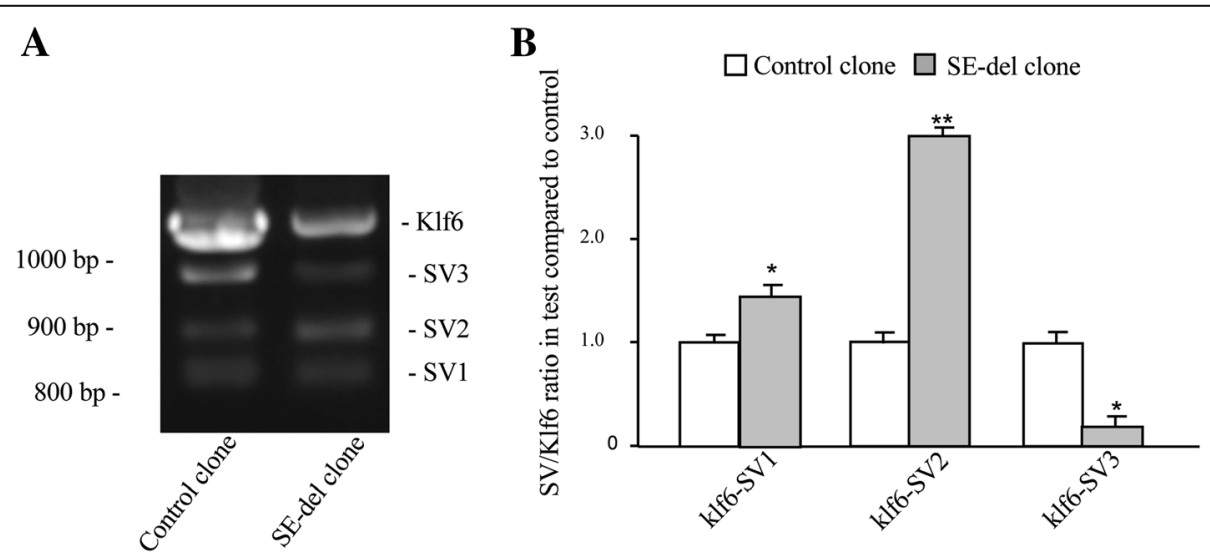

Fig. 2 Expression of Klf6-SV isoforms in the target clones. a RT-PCR of CDNAs with Klf6 variant-specific primers [9]. PCR products were visualized after agarose gel electrophoresis and ethidium bromide staining. b SV/KIf6 mRNA ratio analysis by qRT-PCR. The figure shows KIf6-SV expression relatively to Klf6 expression in the target clones. The results are the mean of three different quantifications, ${ }^{*} p<0.05,{ }^{* *} p<0.01$ 
A

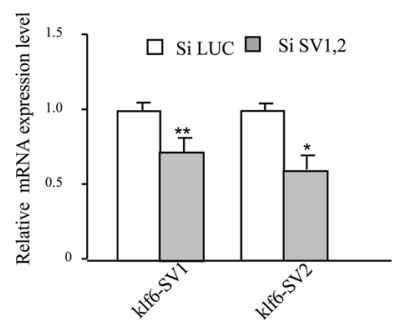

C

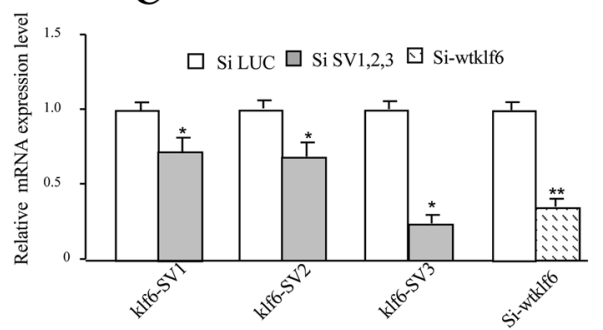

B

D
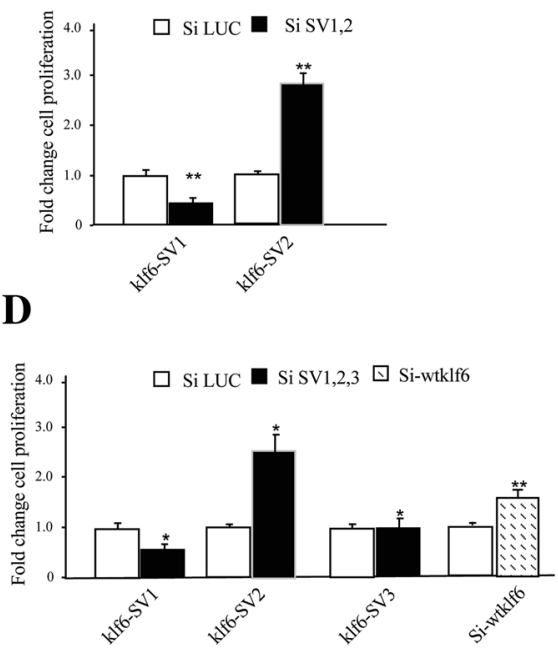

Fig. 3 Expression of KIf6-SV isoforms and cell proliferation assay in the target clones. a Expression assay of KIf6-SV1 and -SV2. The SE-del clone cells were transfected with pSuper-siSV1, 2 or control pSuper-siluc. b Cell proliferation assay. [3H] thymidine incorporation was determined at 72 h. Each experiment was performed in triplicate, ${ }^{* *} p<0.01$. c Expression assay of KIf6-SV isoforms: The SE-non-del clone cells were transfected with pSuper-siSV1,2,3 or control pSuper-siLuc. The SE-non-del clone cells were transfected with pSuper-si-wtKIf6 or control pSuper-siLuc. The results represent the mean of three amplifications, ${ }^{* *} p<0.01,{ }^{*} p<0.05$. $\mathbf{d}$ Cell proliferation assay. Each experiment was performed in triplicate, ${ }^{* *} p<0.01,{ }^{*} p<0.05,{ }^{*} p>0.05$

by Western blot (Fig. 4b). A similar method was used to elucidate the apoptotic mechanism induced by over-expression of Klf6-SV2. Among the proteins involved in apoptosis, elevated expression of the proapoptotic Bcl-2-associated Bax gene was demonstrated (Fig. 4c). Increased expression in Bax was also confirmed by Western blotting (Fig. 4b), and the results suggested that Klf6-SV2-induced apoptosis is associated with the mitochondrial apoptotic pathway. Knockdown of Klf6-SV2 expression by siRNA (Fig. 4a $(+)$, c (+)) induces inhibition of $p 21$ and Bax at the protein (Fig. 4b) and mRNA (Fig. 4a, c). As a result, Klf6-SV2 exhibits partially mediated anti-proliferative and pro-apoptotic effects through induction of $p 21$ (CIP / WAF1) and Bax.

Knockdown of Klf6 variants by various siRNAs in SEnon-del clone can be observed in which over-expression of Klf6-SV2 increases the expression of $p 21$ and $B a x$ at the protein level (Fig. 5c) and mRNA (Fig. 5a, b). In addition, over-expression of various variants in SE-nondel clone also demonstrated that increased expression of $p 21$ and Bax are dependent on over-expression of Klf6SV2 (Fig. 5d-f]. As shown in Fig. 5, unlike the other variants, Klf6-SV3 did not affect the expression of $P 21$ and $B a x$ genes $(\# p>0.05)$. Upregulation of Klf6-SV1 reduces the expression of $P 21$ and Bax genes, but its
A

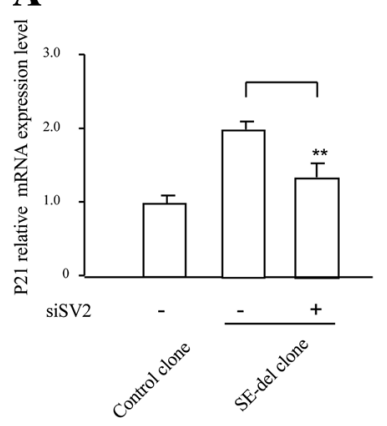

B

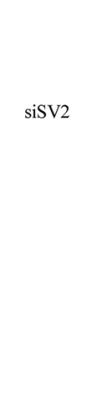

C

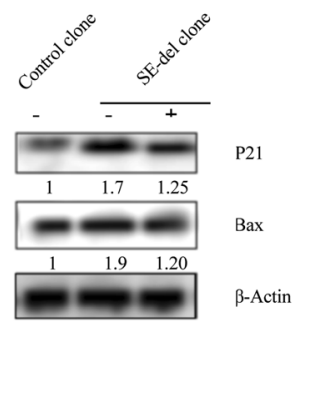

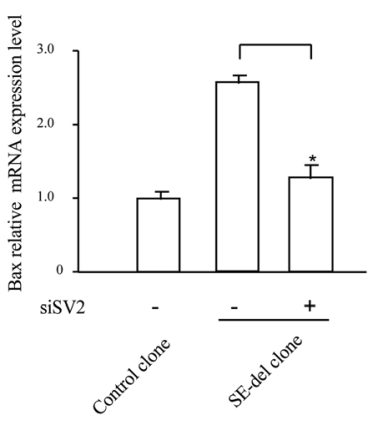

Fig. 4 Over-expression of KIf6-SV2 triggers increased expression of p21 and Bax. a Expression of p21 mRNA in SE-del clone transfected with siSV2. The results represent the mean of three amplifications, ${ }^{* *} p<0.01$. b Expression of $p 21$ and Bax proteins in SE-del clone transfected with siSV2. The gels are representative of three analyses. $\mathbf{c}$ Expression of Bax mRNA in SE-del clone transfected with siSV2. The results represent the mean of three amplifications, ${ }^{*} p<0.05$ 

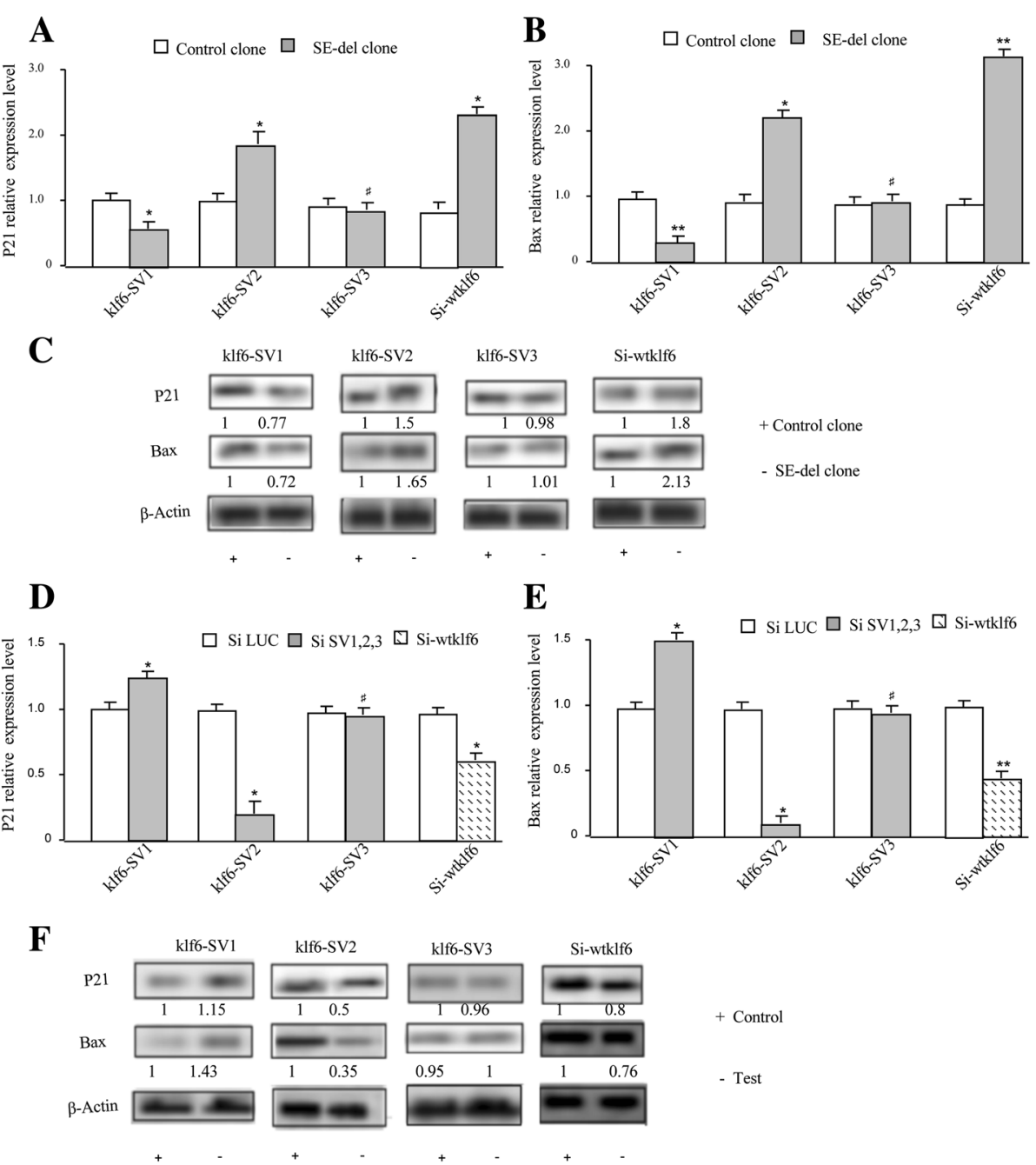

Fig. 5 The effect of Klf6 variants on the expression of $P 21$ and Bax genes. a Expression of p21 mRNA in SE-non-del clone transfected with various siRNA. The results represent the mean of three amplifications, ${ }^{*} p>0.05,{ }^{*} p<0.05$. $\mathbf{b}$ Expression of Bax mRNA in SE-non-del clone transfected with various siRNA. The results represent the mean of three amplifications, ${ }^{* *} p<0.01,{ }^{*} p<0.05,{ }^{*} p>0.05$. c Expression of $p 21$ and Bax proteins in SEnon-del clone transfected with various siRNA. $\mathbf{d}$ Expression of $p 21$ mRNA in SE-non-del clone with over-expression of various KIf6 variants. The results represent the mean of three amplifications, ${ }^{*} p>0.05,{ }^{*} p<0.05$. e Expression of Bax mRNA in SE-non-del clone with over-expression of various KIf6 variants. The results represent the mean of three amplifications, ${ }^{* *} p<0.01,{ }^{*} p<0.05,{ }^{\#} p>0.05$. $\mathbf{f}$ Expression of $p 21$ and Bax proteins in SE-non-del clone with over-expression of various Klf6 variants

downregulation increases expression of $P 21$ and $B a x$ genes.

The results show that Klf6-related super enhancer leads to an induction of the cell cycle-regulating $p 21$ and the pro-apoptotic $B a x$ genes mediated by up-regulation of $S V 2$ variant.

\section{Discussion}

The Klf6 transcription factor is closely related to tumorigenesis through high somatic mutation rates in multiple carcinomas including prostate, colon, stomach, liver, lung, and neck $[2,3]$. The role of dysregulated alternative splicing in cancer is now being studied in a variety of cancers, and it is confirmed that many cancer-related genes are alternatively spliced and their expression leads to the production of multi-splice variants with antagonistic function. Klf6 is alternatively spliced into biologically active isoform called Klf6-SV1, $-S V 2$, and -SV3 [4, 5]. Although the functional outcomes of Klf6 splice variants have become clearer, the pathways regulating expression of Klf6 splice variants remain obscure. Therefore, the observation of expression levels of Klf6 variants and their respective functions in human HepG2 cells is essential for further understanding of their mechanisms. Disruption of Klf6-related SE induces inhibition of proliferation in human HepG2 cells. We hypothesized that inhibition of cell proliferation 
induced by the disruption of Klf6-related super enhancer may be associated with Klf6 variants. In our study, the results show that Klf6-related super enhancer regulates expression of Klf6 variants in human HepG2 cells. As shown in Fig. 2b, the expression of Klf6-SV2 was three times higher than one of the control, but the expression of Klf6-SV1 was increased very little. Specifically, the functional modeling of findings using siRNA specific to Klf6-SV2 in human HepG2 cells revealed that changes in expression level directly affect cell proliferation. We conducted an experiment to determine whether the over-expression of Klf6-SV2 induced by disruption of Klf6-related SE is actually associated with cell proliferation in human HepG2 cells. As shown in Fig. 3, down-expression of $K l f 6-S V 1,-S V 2$, and $-S V 3$ inhibited cell proliferation in siSV1 cells and promoted in siSV2 cells, but not in siSV3 cells. Furthermore, cell proliferation was shown to be directly associated with Klf6-SV2 levels. These results show that over-expression of Klf6-SV2 induced by disruption of Klf6-related SE is an important factor associated with inhibition of cell proliferation in human HepG2 cells. The above results are further supported by literature $[1,7,9,12,13,23]$ that overexpression of Klf6-SV1 promotes cell proliferation, while over-expression of Klf6-SV2 inhibits cell proliferation. It is also consistent with data that downregulation of wtKlf6 promotes cell proliferation in hepatocellular carcinoma (HCC) [24]. We demonstrated that over-expression of Klf6-SV2 induces transcriptional activation of $p 21$ (CIP/ WAF1) and Bax genes (Fig. 5). The results show that overexpression of Klf6-SV2 exerts partially mediated antiproliferative and pro-apoptotic effect by induction of $p 21$ (CIP / WAF1) and Bax. The results are consistent with the literature $[1,9,12,13,23]$ showing that over-expression of Klf6-SV2 induces anti-proliferation and apoptosis.
We proposed a novel model regulating cell proliferation via modulation of Klf6-SV2 expression into the growth-relating isoforms (Fig. 6). Klf6-related super enhancer regulates expression of Klf6-SV2, thereby altering the relative ratio of Klf6 to Klf6-SV2, which leads to inhibition of cell proliferation. This model requires further studies, such as what is the signaling pathway between the super enhancer and biologically active isoforms, and how it is regulated [25-29].

\section{Conclusions}

Several studies have identified new cancer therapeutics that target super-enhancers of various tumor types. In our study, we have elucidated the functional properties of the Klf6-related super enhancer on the expression of Klf6 splice variants which may be helpful in the target treatment of Klf6-related super enhancer. the disruption of Klf6-related super enhancer showed not only induced the up-regulation of Klf6-SV2 but also led to a significant reduction of proliferation in HepG2 cells. It was demonstrated that the disruption of Klf6-related super enhancer led to the induction of $p 21$ and $B a x$ genes mediated by the up-regulation of Klf6-SV2. Therefore, it showed that Klf6-related super enhancer modulates cell proliferation via the regulation of Klf6-SV2 expression in human hepatoma (HepG2) cells. The results provide the functional significance of Klf6-related super enhancer in understanding the transcriptional regulation mechanism of Klf6.

But this result raises an interesting question of how cellular signals converge to regulate splicing events in promoting tumor progression. Targeted analysis of molecular pathways of Klf6-related super enhancer that regulates Klf6-SV2 expression will provide further insight into the role of this gene in the progression of human cancer.
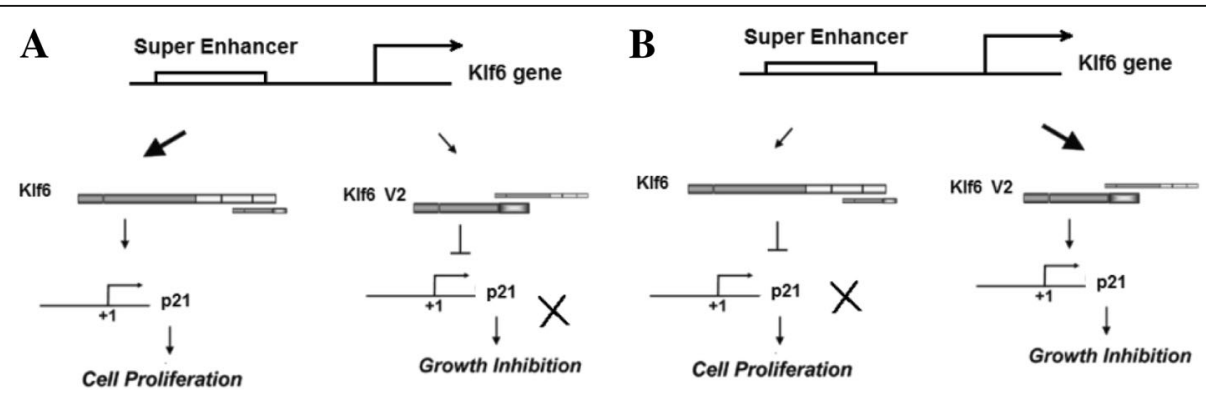

Fig. 6 The model regulating cell proliferation by super enhancer. a Pre-disruption model of KIf6-related SE. When KIf6-related SE is not disrupted, it upregulates KIf6 expression and induces cellular proliferation. b Post-disruption model of KIf6-related SE. When KIf6-related SE is disrupted, it upregulates KIf6-SV2 expression which leads to p21-mediated growth inhibition. The size of the arrow in the figure represents the relative expression intensity of the target gene. In other words, an arrow indicated by a thick line means strong gene expression compared to a thinly marked arrow 


\section{Abbreviations}

Klf6: Kruppel-like factor 6; wtKlf6: Wild-type Klf6; qRT-PCR: Quantitative realtime PCR; WT: Wild type; SEs: Super enhancers; SE-del clones: SEs deleted clone; SE-non-del clone: SE-non-deleted clone; siRNA: Small interfering RNA; Luc construct: Luciferase constructs

\section{Acknowledgements}

Not applicable.

\section{Authors' contributions}

RKC was responsible for the overall project design and experiments, and KC was involved in the transfection experiments of HepG2. CSI was engaged in cell proliferation assays and Western blot experiments. SJH and OSH performed Klf6-SV1, -SV2, and -VS3 expression experiments. All authors have read and approved the manuscript.

\section{Funding}

Not applicable.

\section{Availability of data and materials}

Not applicable.

\section{Ethics approval and consent to participate}

Not applicable.

\section{Consent for publication}

Not applicable.

\section{Competing interests}

The authors declare that they have no competing interests.

\section{Author details}

${ }^{1}$ School of Management, Harbin Institute of Technology, Harbin, China. ${ }^{2}$ Life Science Department, University of Science, Pyongyang, Democratic People's Republic of Korea. ${ }^{3}$ Life Science Department, Kim IL Sung University, Pyongyang, Democratic People's Republic of Korea. ${ }^{4}$ Chemistry Department, University of Science, Pyongyang, Democratic People's Republic of Korea.

Received: 26 June 2019 Accepted: 12 July 2019

\section{Published online: 08 October 2019}

\section{References}

1. Difeo A, Huang F, Sangodkar J, Terzo EA, Leake D, Narla G, Martignetti JA (2009) Klf6-SV1 is a novel antiapoptotic protein that targets the BH3-only protein NOXA for degradation and whose inhibition extends survival in an ovarian cancer model. Cancer Res 69:4733-4741

2. Banck MS, Beaven SW, Narla G, Walsh MJ, Friedman SL, Beutler AS (2006) KIf6 degradation after apoptotic DNA damage. FEBS Letters 580:6981-6986

3. Chen C, Hyytinen E-R, Sun X, Helin HJ (2003) Deletion, mutation, and loss of expression of KLF6 in human prostate cancer. Am J Pathol 162:1349-1354

4. Benzeno S, Narla G, Allina J, Cheng GZ, Reeves HL, Banck MS (2004) Cyclin-dependent kinase inhibition by the KIf6 tumor suppressor protein through interaction with cyclin D1. Cancer Res 64:3885-3891

5. Reeves HL, Narla G, Ogunbiyi O, Haq Al, Katz A, Benzeno S (2004) Kruppel-like factor 6 (Klf6) is a tumor-suppressor gene frequently inactivated in colorectal cancer. Gastroenterology 126:1090-1103

6. Kremer-Tal S, Reeves HL, Narla G, Thung SN, Schwartz M, Difeo A (2004) Frequent inactivation of the tumor suppressor Kruppel-like factor 6 (KIf6) in hepatocellular carcinoma. Hepatology 40:1047-1052

7. DiFeo A, Martignetti JA, Narla G (2009) The role of KLF6 and its splice variants in cancer therapy. Drug Resist Updat 12:1-7

8. Liang W-C, Wang $y$, Xiao L-J, Wang y-B (2014) Identification of miRNAs that specifically target tumor suppressive KIf6-FL rather than oncogenic KIf6-SV isoform. RNa Biol 11(7):845-854

9. Narla G, Difeo A, Reeves HL (2005) A germline DNA polymorphism associated with increased prostate cancer risk enhances alternative splicing of the KLF6 tumor suppressor gene. Cancer Res 65:1213-1222

10. Racca AC, Ridano ME, Bandeira CL, Bevilacqua E, Avvad Portari E, Genti-Raimondi S, Graham CH, Panzetta-Dutari GM (2016) Low oxygen tension induces Krüppel-Like Factor 6 expression in trophoblast cells. Placenta 45:50-57
11. Humbert M, Halter V, Shan D, Laedrach J, Leibundgut EO, Baerlocher GM, Tobler A, Fey MF, Tschan MP (2011) Deregulated expression of Kruppel-like factors in acute myeloid leukemia. Leuk Res 35:909-913

12. Hanoun N, Bureau C, Diab T (2010) The SV2 variant of Klf6 is down-regulated in hepatocellular carcinoma and displays anti-proliferative and pro-apoptotic functions. J Hepatol 53:880-888

13. D'Astolfo DS, Gehrau RC, Bocco JL, Koritschoner NP (2008) Silencing of the transcription factor Klf6 by siRNA leads to cell cycle arrest and sensitizes cells to apoptosis induced by DNA damage. Cell Death Differ 15:613-616

14. Sirach E, Bureau C, Peron JM, Pradayrol L, Vinel JP, Buscail L, Cordelier P (2007) Klf6 transcription factor protects hepatocellular carcinoma-derived cells from apoptosis. Cell Death Differ 14:1202-1210

15. Jakob L'n, Hoke HA, Lin CY, Lau A, Orlando DA, Vakoc CR, Bradner JE, Lee TI, Young RA (2013) Selective inhibition of tumor oncogenes by disruption of super enhancers. Cell 153:320-334

16. Mansour MR, Abraham BJ, Anders L, Berezovskaya A, Gutierrez A, Durbin AD Etchin J, Lawton $L$ (2014) An oncogenic super-enhancer formed through somatic mutation of a noncoding intergenic element. Science 346:6215

17. Bannikov AV, Lavrov AV (2017) CRISPR/CAS9, the king of genome editing tools. Mol Biol 51(4):514-525

18. Pougach KS, Lopatina AV, Severinov KV (2012) CRISPR adaptive immunity systems of prokaryotes. Mol Biol 46(2):175-182

19. Zhigalova NA, Zhenilo SV, Artemov AV, Prokhortchouk EB (2017) CRISPR/Cas9-editing-based modeling of hypoxia in renal cancer cells. Mol Biol 51(5):728-732

20. Ri KC, Kim KC, Kong SH, Ri JH (2018) The disruption of Klf6-related super-enhancer induces growth inhibition and apoptosis in human HepG2 Cells. Genet Mol Res 17(1):gmr16039888

21. Ri KC, Kim JS, Kim C (2017) Identification ofKlf6-related super enhancer in human hepatoma (HepG2) cells by CRISPR technique. Genet Mol Res 16(4): gmr16039841

22. Brummelkamp TR, Bernards R, Agami R (2002) A system for stable expression of short interfering RNAs in mammalian cells. Science 296:550-553

23. Hatami R, Sieuwerts AM (2013) KLF6-SV1 drives breast cancer metastasis and is associated with poor survival. Sci Transl Med 5(169):169ra12

24. Kremer-Tal S, Narla G (2007) Downregulation of KLF6 is an early event in hepatocarcinogenesis, and stimulates proliferation while reducing differentiation. J Hepatol 46:645-654

25. Cong L, Ran FA, Cox D, Lin S, Barretto R (2013) Multiplex genome engineering using CRISPR/Cas systems. Science 339:819-823

26. Didych DA, Tyulkina DV, Pleshkan W, Alekseenko IV, Sverdlov ED (2015) Are super-enhancers regulators of regulatory genes of development and cancer. Mol Biol 49(6):818-824

27. Denes Hnisz, Brian J. Abraham, Tong Ihn Lee, Ashley Lau, Violaine Saint-Andre', Alla A. Sigova, Heather A. Hoke and Richard A. Young (2013) Super-enhancers in the control of cell identity and disease. Cell 155: 934-947.

28. Hnisz D, Schuijers J, Lin CY, Weintraub AS, Abraham BJ, Lee TI, Bradner JE, Young RA (2015) Convergence of developmental and oncogenic signaling pathways at transcriptional super-enhancers. Mol Cell 58:1-9

29. Narla G, DiFeo A, Yao S, Banno A (2005) Targeted Inhibition of the Klf6 splice variant, KIf6 SV1, suppresses prostate cancer cell growth and spread. Cancer Res 65(13):5761-5768

\section{Publisher's Note}

Springer Nature remains neutral with regard to jurisdictional claims in published maps and institutional affiliations. 